\title{
Expression of long non-coding RNAs in chondrocytes from proximal interphalangeal joints
}

\author{
DONG LV*, CHANGZHENG SU* ${ }^{*}$ ZHEN LI, XINGYU CHAI, ZHENGWEN XU and TAO PANG \\ Department of Orthopedics, Tengzhou Central People's Hospital, Tengzhou, Shandong 277500, P.R. China
}

Received August 19, 2016; Accepted May 12, 2017

DOI: $10.3892 / \mathrm{mmr} .2017 .7274$

\begin{abstract}
Osteoarthritis (OA) of hand is a common disease, resulting in disability of the hands. The pathogenesis of hand $(\mathrm{H})$ OA remains to be elucidated, and findings from knee and hip joints cannot be simply applied to HOA. To improve knowledge on the specific biology and pathobiology of HOA, the present study performed bioinformatics analyses to analyze the long non-coding (lnc) RNA expression profile in human chondrocytes of proximal interphalangeal (PIP) finger joints and knee joints. Gene expression data were downloaded from the Gene Expression Omnibus database, and PIP and knee chondrocytes were analyzed ( $n=3 /$ group). Probes of the Affymetrix Human Gene 2.0 ST Microarray were annotated to obtain information about lncRNA expression profile. Compared with chondrocytes from knee joints, chondrocytes derived from PIP joints had significantly different lncRNA expression profiles, and 1,172 IncRNAs were differentially expressed. Compared with chondrocyte from knee joints, 534 lncRNAs were upregulated and 638 lncRNAs were downregulated in chondrocytes from PIP joints. A co-expression network was constructed to analyze the correlation between lncRNAs and protein-coding genes. Function annotation analyses suggested that protein-coding genes that are co-expressed with IncRNAs are enriched in the biological processes of bone morphogenesis, bone development and cartilage development. In conclusion, the present study demonstrated that chondrocytes derived from PIP joints exhibit a significant difference in lncRNA expression compared with chondrocytes derived from knee joints.
\end{abstract}

Correspondence to: Dr Tao Pang, Department of Orthopedics, Tengzhou Central People's Hospital, 181 Xingtan Road, Tengzhou, Shandong 277500, P.R. China

E-mail: doctor_pt@yeah.net

${ }^{*}$ Contributed equally

Abbreviations: HOA, hand osteoarthritis; lncRNA, long non-coding RNA; PIP, proximal interphalangeal

Key words: hand osteoarthritis, chondrocytes, lncRNA, expression, proximal interphalangeal joints

\section{Introduction}

Osteoarthritis (OA) is a comment disease that affects many major joints including the hip, spine, knee and the hand $(1,2)$. OA leads to severe functional and emotional burdens to patients and causes heavy economic burdens (3). Hand osteoarthritis (HOA) is one of the most common kinds of OA and HOA is a leading cause of disability of the hands. The incidence of HOA is $\sim 20 \%$ in people aged 65 or older (4).

$\mathrm{HOA}$ is considered as a primarily degenerative process with inflammation (5), and degradation of articular cartilage is the hallmark of HOA. The cartilage degradation process of the knee and hip joints has been well investigated using animal models or human chondrocyte culture. However, the molecular mechanism of HOA is largely unknown. Limited by different anatomical shapes and mechanical requirements, findings from knee or hip joints cannot be simply applied to other joints. For HOA, Stradner et al (6) successfully established a chondrocyte culture of proximal interphalangeal (PIP) cartilage, and demonstrated that chondrocytes from PIP and knee joints have distinct expression patterns of protein-coding genes.

Previous evidence has indicated that non-coding RNAs serve important roles in various biological processes including carcinogenesis, cell differentiation, metabolism, and immunity responses (7-12). Long non-coding RNA (lncRNA) is a kind of non-coding RNA transcript, which is $>200 \mathrm{nt}$ long and does not encode proteins (7). Researchers have demonstrated that lncRNAs are involved in regulating the expression levels of various factors involved in the pathological process of OA, including maternally expressed 3 (13), imprinted maternally expressed transcript (14) and HOX transcript antisense RNA (15). However, the IncRNA expression profile from hip and knee joints cannot be simply applied to HOA, and the expression pattern of IncRNA in HOA remains unknown. Therefore, the present study performed a bioinformatics analysis to systematically analyze lncRNA expression profile in the human cartilage of the PIP finger joints by annotation of microarray data to improve the understanding about the pathology of HOA.

\section{Materials and methods}

Dataset selection. The gene expression data used in the current study was obtained from the publicly available Gene Expression Omnibus (GEO) database (www.ncbi.nlm.nih. gov/geo/). This GSE68038 dataset was identified and analyzed, 
which contains 3 samples of knee chondrocytes and 3 samples of PIP chondrocytes. The GSE68038 dataset was based on the Affymetrix Human Gene 2.0 ST Array [transcript (gene) version] (Affymetrix; Thermo Fisher Scientific, Inc.). The raw CEL files were downloaded and quantile normalized and background adjusted using Robust Multichip Average (Windows version) software v1.20.0 (16). After normalization, the expression value of each probe was obtained. The normalized data were then analyzed with Significant Analysis of Microarray software v4.01 (17). Unpaired t-test was used to calculate differentially expressed genes and $\mathrm{P}<0.05$ was considered to indicate a statistically significant difference. Differentially expressed IncRNAs were clustered and visualized by Cluster 3.0 software (http://bonsai.hgc.jp/ mdehoon/software/cluster/software.htm).

Annotation of lncRNA probes. To identify probe sets that target lncRNA transcripts, the BioMart data portal (www.biomart.org/) was used to download annotations of lncRNA probes. Following this, the probe sets were filtered according to Ensemble transcript type, and transcripts with 'antisense', 'processed_transcripts', 'lincRNA', 'non_sense mediated_decay', 'sense intronic' and 'sense overlapping' were selected as lncRNA $(18,19)$. After creation of annotation file, the differentially probe sets were matched and annotated.

Co-expression network construction. The co-expression network of lncRNA protein-coding genes was built to identify the interactions between coding genes and lncRNA (20). Differentially expressed lncRNAs and protein-coding genes with $\mathrm{P}<0.01$ were used to construct the co-expression network, and the normalized expression values were retrieved. For each pair of coding gene-lncRNA, coding-coding genes or lncRNA-lncRNA, the $\mathrm{P}$ value of Pearson correlation and significant correlation pairs $(\mathrm{P}<0.05)$ were used to construct the network (21). Cytoscape software v3.3.0 was utilized to visualize the co-expression network (www.cytoscape.org/).

Functional enrichment analysis. The gene functional enrichment analysis was performed by the Database for Annotation, Visualization and Integrated Discovery online tools (http://david.ncifcrf.gov/) (22). $\mathrm{P}<0.05$ and false discovery rate $<0.05$ was considered as significant enrichment.

\section{Results}

Gene expression profiles of chondrocytes were analyzed with Affymetrix Human Gene 2.0 ST Arrays. After annotation, $\sim 18,000$ probes mapped to IncRNAs were identified. According to the statistical threshold, 1,172 differentially expressed IncRNAs were identified. Compared with chondrocytes from knee joints, 534 lncRNAs were upregulated and 638 lncRNAs were downregulated in chondrocytes from PIP joints. The expression patterns of lncRNAs were different between chondrocytes from knee and PIP joints, and the top 100 differentially expressed $\operatorname{lncRNAs}$ are presented as a heat map (Fig. 1). As presented, a set of lncRNAs were significantly differentially expressed between PIP and knee joints. The detailed information of 20 most upregulated and downregulated lncRNAs are presented in Table I. Compared with knee joints chondrocyte, ENST00000451530 was mostly upregulated in

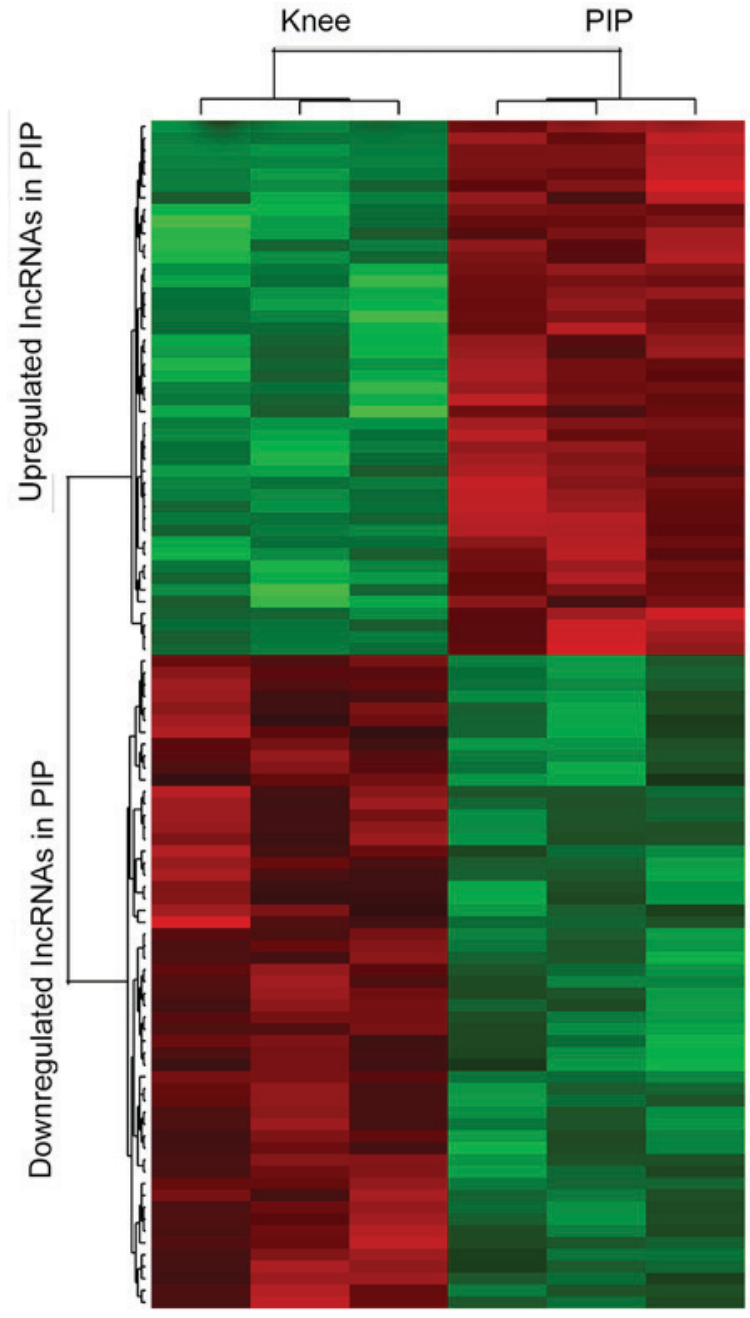

Figure 1. Heat map of differentially expressed lncRNAs between chondrocytes from PIP and knee joints. Each row represents an lncRNA and each column represents a sample, the left 3 columns are chondrocytes from knee joints and the right 3 columns are chondrocytes from PIP joints; red, upregulated lncRNAs, green, downregulated lncRNAs. lncRNA, long non-coding RNA; PIP, proximal interphalangeal.

chondrocytes from PIP, and ENST00000462445 was mostly downregulated.

Co-expression networks of lncRNA and protein-coding genes may provide information to infer the biological function of lncRNA, as genes in the same signaling pathway or with the same function may have similar expression patterns. Additionally, co-expression networks have been widely used to predict the potential biological function of lncRNAs. Therefore, a co-expression network was built between lncRNA and protein-coding genes. As presented in Fig. 2A, IncRNAs and protein-coding genes are closely connected. In the co-expression network, RP11-6E9.4 (ENST00000508955) and RP11-713P17.3 (ENST00000529070) were 2 lncRNAs that co-expressed with $>30$ protein-coding genes. In addition, many inflammation-associated genes were co-expressed with these lncRNAs, including interleukin (IL)-7R, IL-19 and chemokine (C-C) motif ligand 1. On the other hand, several Wnt pathway genes, including Wnt5A, were also co-expressed with these lncRNAs.

Co-expression networks are a potential method to predict lncRNA function, as genes involved in the same biological 


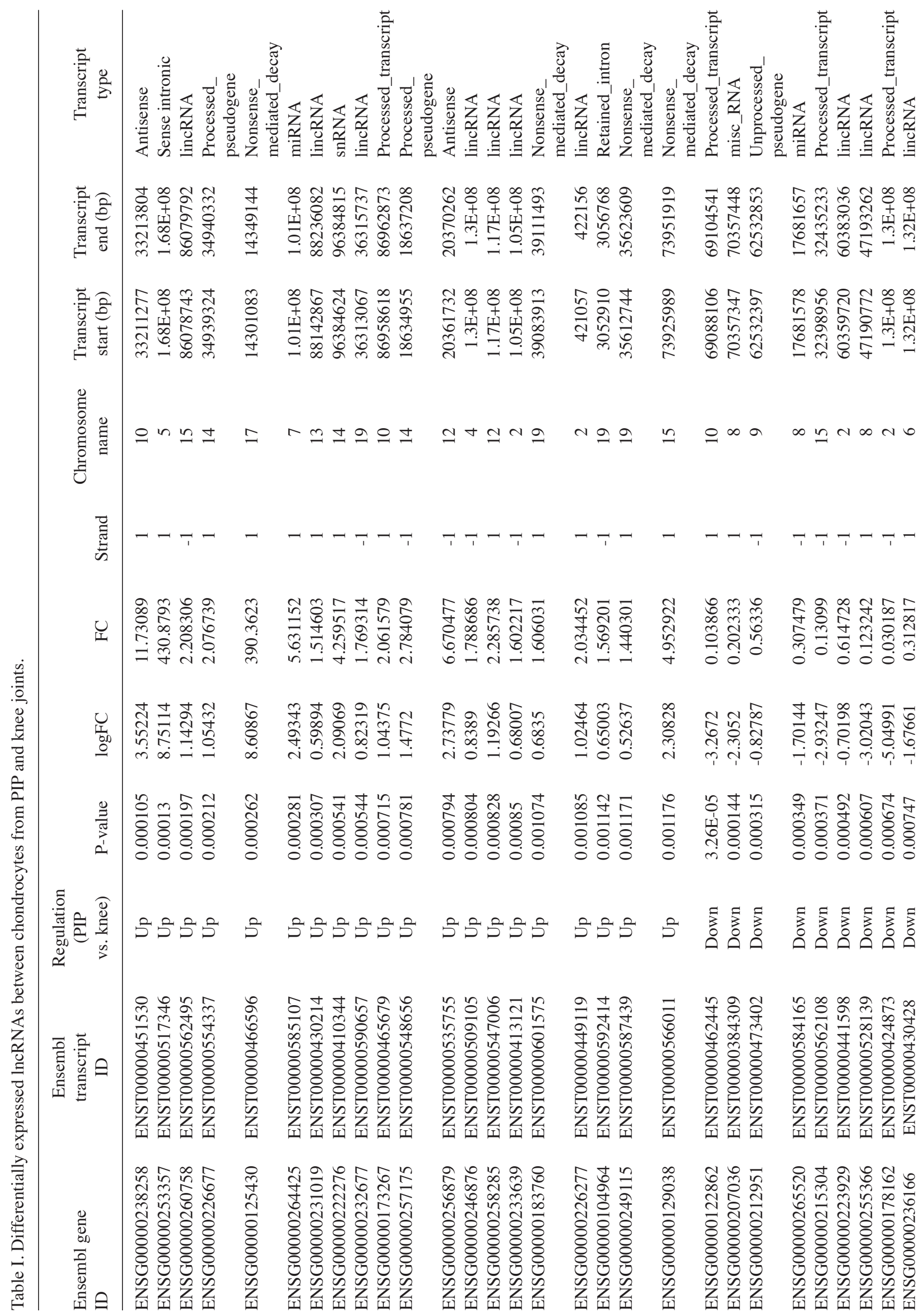




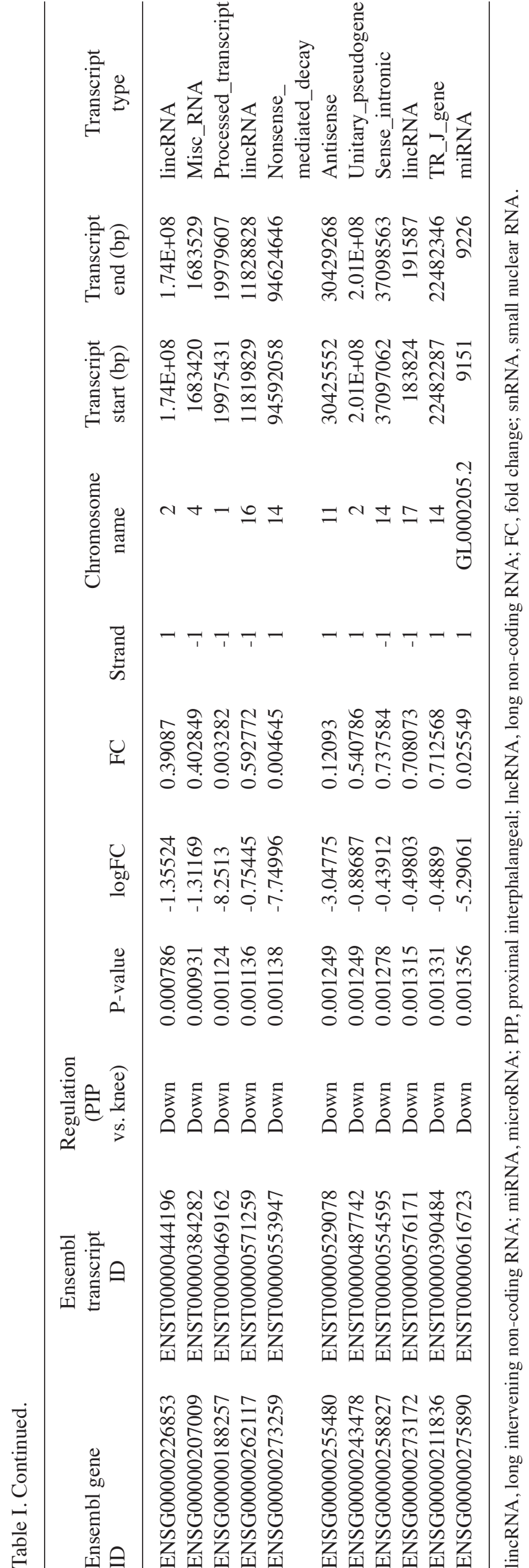

processes are usually co-expressed. Therefore, a functional enrichment analyses for protein-coding genes in the co-expression network was performed to further predict their biological functions. As presented in Fig. 2C and D, gene ontology analyses demonstrated that these protein-coding genes were significantly associated with bone morphogenesis, bone development, skeletal system development and cartilage development, suggesting these protein-coding genes and lncRNAs may be involved in OA pathogenesis.

\section{Discussion}

Articular cartilage degradation is a hallmark of OA. Human chondrocyte culture has been extensively used to investigate the cartilage degradation of knee and hip joints. However, little is known is about the cartilage degradation in HOA, and findings from hip and knee joints cannot be simply applied to HOA due to different anatomical shapes and mechanical requirements between joints $(23,24)$.

The present study compared lncRNA expression profiles between chondrocytes from PIP and knee joints. The results demonstrated that chondrocytes from PIP and knee joints have different lncRNA expression patterns. Compared with chondrocytes from knee joints, 534 lncRNAs were upregulated and 638 lncRNAs were downregulated in chondrocytes from PIP joints. The different expression profile of IncRNA between chondrocyte from PIP and knee joints supported previous reports that IncRNA expression is highly temporally and specially specific (7). Therefore, these differentially expressed lncRNAs in PIP joints may be involved in the biological function of chondrocytes, and the pathological process of HOA.

Previous research has investigated the lncRNA expression profile in patients with rheumatoid arthritis $(25,26)$, and it was concluded that IncRNA may contribute to the pathogenesis of rheumatoid arthritis (26). Compared with these studies in rheumatoid arthritis, IncRNA expression in HOA was analyzed with bioinformatics analyses, and the potential association between IncRNAs and coding genes was inferred. However, HOA and rheumatoid arthritis have different pathologies and clinical features; therefore, findings between the two cannot be easily compared.

Co-expression networks between IncRNA and proteincoding genes were constructed to infer the potential function of these lncRNAs. GO enrichment analyses demonstrated that protein-coding genes in the co-expression network are associated with many skeletal system-specific items, including bone, cartilage and skeletal system development. The results suggested that these lncRNAs were highly likely involved in these biological processes, and these lncRNAs may also serve important roles in the pathological process of HOA.

Subsequently, the present study demonstrated that RP11-6E9.4 (ENST00000508955) and RP11-713P17.3 (ENST00000529070) were co-expressed with the most protein-coding genes. Compared with chondrocytes from knee joints, RP11-6E9.4 and RP11-713P17.3 were significantly downregulated in PIP joints. In addition, we constructed a co-expression network of coding genes and lncRNAs and found RP11-6E9.4 and RP11-713P17.3 were co-expressed with several inflammation and cartilage specific genes, including 
A

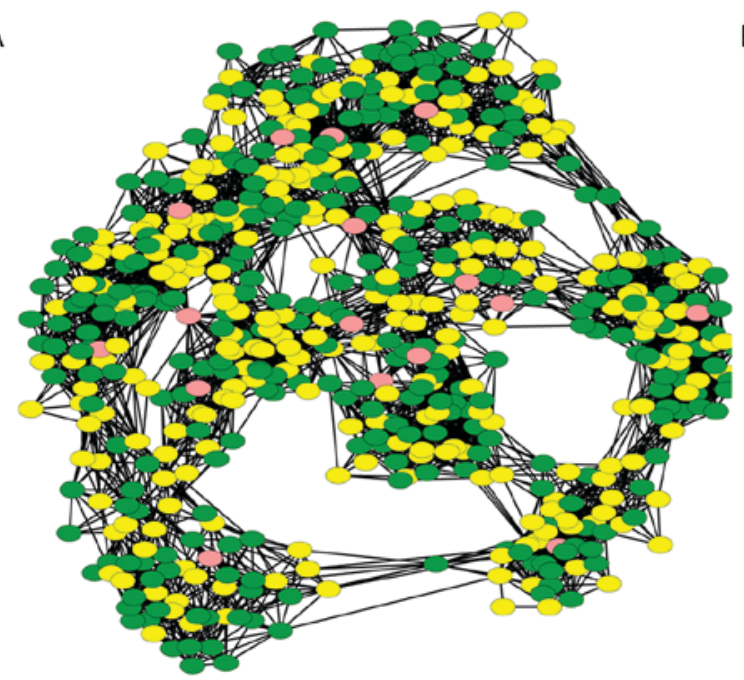

B

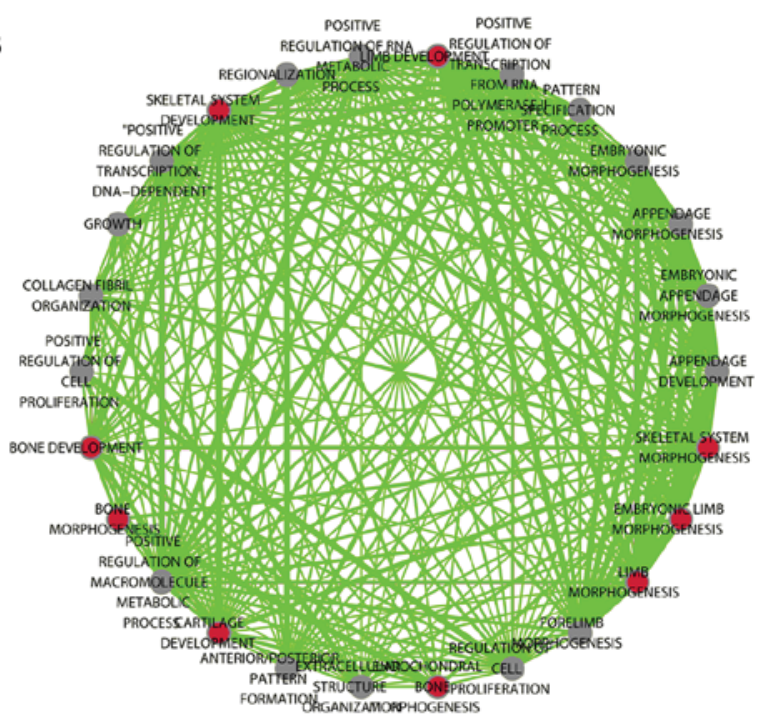

C

Fold enrichment

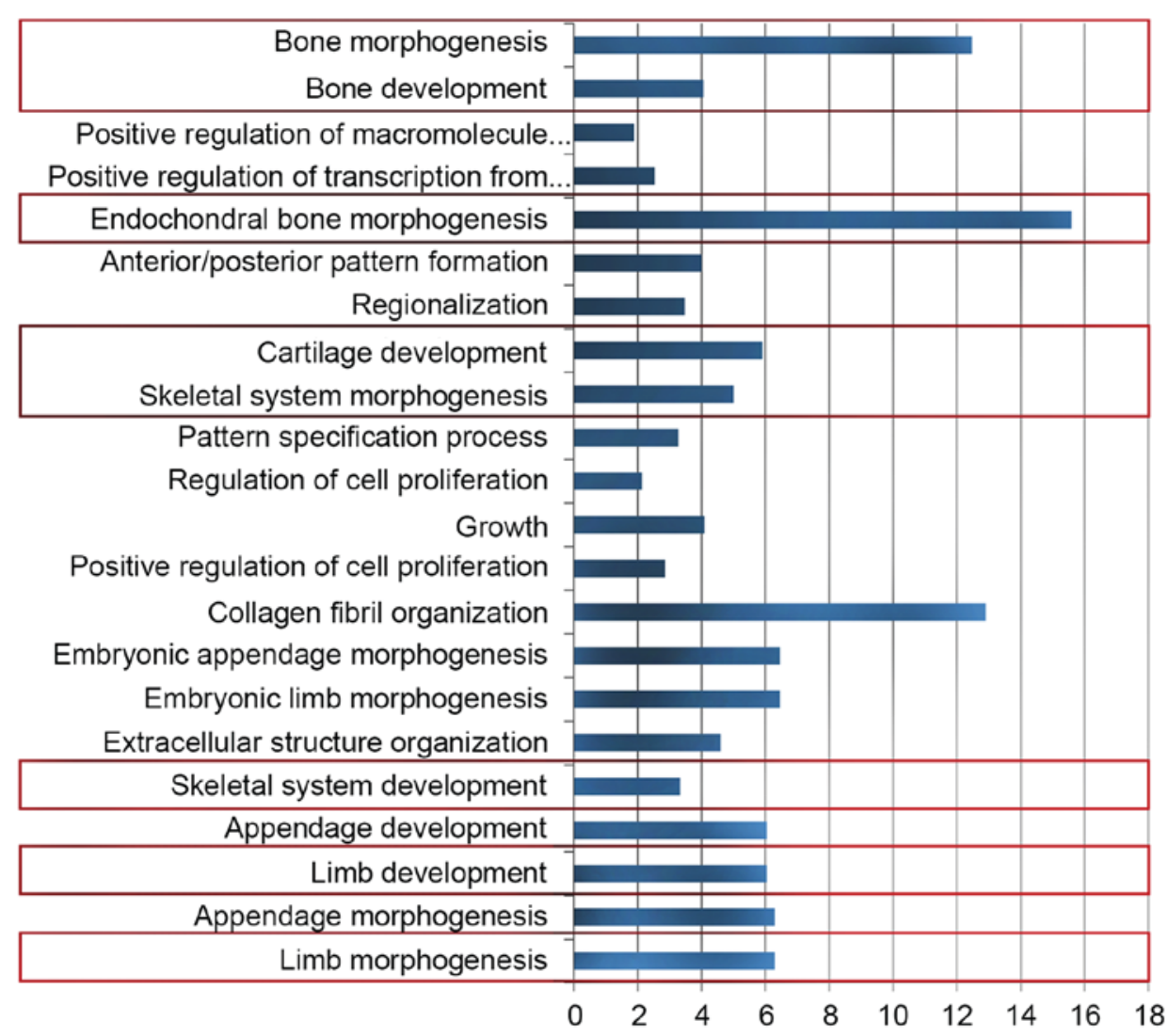

Figure 2. (A) Co-expression network of lncRNAs and protein-coding genes. Yellow dots, lncRNAs; green dots, protein-coding genes; light red dots, centers of the sub-networks. (B) Enrichment plot of gene ontology results. Biological processes associated with bone and cartilage are highlighted with red, and grey dots are terms not associated with bone or cartilage. (C) Column chart of gene ontology results, and bone and cartilage-associated gene ontology terms are highlighted with red boxes. lncRNA, long non-coding RNA.

collagen type I alpha I and transcription factor SOX9 (27). Therefore, RP11-6E9.4 and RP11-713P17.3 are potentially involved in the pathogenesis of OA; however, further experiments are required to validate the functional roles of these lncRNAs.

The biological function and molecular mechanism have been widely investigated in various biological and pathological processes. To the best of our knowledge, there is currently no literature on the expression profile of HOA in lncRNA. However, the present study has several limitations. Firstly, this is only a bioinformatics analysis; thus, these findings should be further validated by laboratory experiments. Secondly, the sample sizes analyzed were small, which might limit the application of these findings.

In conclusion, the present study demonstrated that chondrocytes from PIP joints have different lncRNA expression profiles. 
These findings may improve knowledge on the biological roles of lncRNA and the pathology of HOA.

\section{References}

1. Battié MC, Videman T and Parent E: Lumbar disc degeneration: Epidemiology and genetic influences. Spine 29: 2679-2690, 2004

2. Kotlarz H, Gunnarsson CL, Fang H and Rizzo JA: Insurer and out-of-pocket costs of osteoarthritis in the US: Evidence from national survey data. Arthritis Rheum 60: 3546-3553, 2009.

3. Hochberg MC: Epidemiology of osteoarthritis: Current concepts and new insights. J Rheumatol Suppl 27: 4-6, 1991.

4. Zhang Y, Niu J, Kelly-Hayes M, Chaisson CE, Aliabadi P and Felson DT: Prevalence of symptomatic hand osteoarthritis and its impact on functional status among the elderly: The Framingham Study. Am J Epidemiol 156: 1021-1027, 2002.

5. Kortekaas MC, Kwok WY, Reijnierse M, Huizinga TW and Kloppenburg M: In erosive hand osteoarthritis more inflammatory signs on ultrasound are found than in the rest of hand osteoarthritis. Ann Rheum Dis 72: 930-934, 2013.

6. Stradner MH, Dreu M, Angerer H, Gruber G, Wagner K, Peischler D, Krischan V, Leithner A, Anderhuber F and Graninger WB: Chondrocyte cultures from human proximal interphalangeal finger joints. J Orthop Res 34: 1569-1575, 2016.

7. Ponting CP, Oliver PL and Reik W: Evolution and functions of long noncoding RNAs. Cell 136: 629-641, 2009.

8. Esteller M: Non-coding RNAs in human disease. Nat Rev Genet 12: 861-874, 2011.

9. Mercer TR, Dinger ME and Mattick JS: Long non-coding RNAs: Insights into functions. Nat Rev Genet 10: 155-159, 2009.

10. Caputa G and Schaffer JE: RNA regulation of lipotoxicity and metabolic stress. Diabetes 65: 1816-1823, 2016.

11. Wang P, Xue Y, Han Y, Lin L, Wu C, Xu S, Jiang Z, Xu J, Liu Q and Cao X: The STAT3-binding long noncoding RNA lnc-DC controls human dendritic cell differentiation. Science 344 : 310-313, 2014.

12. Kretz M, Siprashvili Z, Chu C, Webster DE, Zehnder A, Qu K, Lee CS, Flockhart RJ, Groff AF, Chow J, et al: Control of somatic tissue differentiation by the long non-coding RNA TINCR. Nature 493: 231-235, 2013

13. Su W, Xie W, Shang Q and Su B: The long noncoding RNA MEG3 Is downregulated and inversely associated with VEGF levels in osteoarthritis. Biomed Res Int 2015: 356893, 2015.

14. Stuhlmüller B, Kunisch E, Franz J, Martinez-Gamboa L, Hernandez MM, Pruss A, Ulbrich N, Erdmann VA, Burmester GR and Kinne RW: Detection of oncofetal h19 RNA in rheumatoid arthritis synovial tissue. Am J Pathol 163: 901-911, 2003.
15. Zhang C, Wang P, Jiang P,Lv Y, Dong C, Dai X, Tan L and Wang Z Upregulation of IncRNA HOTAIR contributes to IL-1 $\beta$-induced MMP overexpression and chondrocytes apoptosis in temporomandibular joint osteoarthritis. Gene 586: 248-253, 2016.

16. Wang P, Lu S, Mao H, Bai Y, Ma T, Cheng Z, Zhang H, Jin Q, Zhao $\mathrm{J}$ and Mao $\mathrm{H}$ : Identification of biomarkers for the detection of early stage lung adenocarcinoma by microarray profiling of long noncoding RNAs. Lung Cancer 88: 147-153, 2015.

17. Maurer MH, Feldmann RE Jr, Brömme JO and Kalenka A: Comparison of statistical approaches for the analysis of proteome expression data of differentiating neural stem cells. J Proteome Res 4: 96-100, 2005.

18. Yang J, Lin J, Liu T, Chen T, Pan S, Huang W and Li S: Analysis of lncRNA expression profiles in non-small cell lung cancers (NSCLC) and their clinical subtypes. Lung Cancer 85: 110-115, 2014.

19. Zhang X, Sun S, Pu JK, Tsang AC, Lee D, Man VO, Lui WM, Wong ST and Leung GK: Long non-coding RNA expression profiles predict clinical phenotypes in glioma. Neurobiol Dis 48: $1-8,2012$

20. Pujana MA, Han JD, Starita LM, Stevens KN, Tewari M, Ahn JS, Rennert G, Moreno V, Kirchhoff T, Gold B, et al: Network modeling links breast cancer susceptibility and centrosome dysfunction. Nat Genet 39: 1338-1349, 2007.

21. Prieto C, Risueño A, Fontanillo C and De las Rivas J: Human gene coexpression landscape: Confident network derived from tissue transcriptomic profiles. PLoS One 3: e3911, 2008.

22. Huang da W, Sherman BT and Lempicki RA: Systematic and integrative analysis of large gene lists using DAVID bioinformatics resources. Nat Protoc 4: 44-57, 2009.

23. Novakofski KD, Berg LC, Bronzini I, Bonnevie ED, Poland SG, Bonassar LJ and Fortier LA: Joint-dependent response to impact and implications for post-traumatic osteoarthritis. Osteoarthritis Cartilage 23: 1130-1137, 2015.

24. Eger W, Schumacher BL, Mollenhauer J, Kuettner KE and Cole AA: Human knee and ankle cartilage explants: Catabolic differences. J Orthop Res 20: 526-534, 2002.

25. Zhang Y, Xu YZ, Sun N, Liu JH, Chen FF, Guan XL, Li A, Wang F, Zhao QF, Wang HY, et al: Long noncoding RNA expression profile in fibroblast-like synoviocytes from patients with rheumatoid arthritis. Arthritis Res Ther 18: 227, 2016.

26. Jiang H, Qin XJ, Li WP, Ma R, Wang T and Li ZQ: LncRNAs expression in adjuvant-induced arthritis rats reveals the potential role of LncRNAs contributing to rheumatoid arthritis pathogenesis. Gene 593: 131-142, 2016.

27. Rogers EL, Reynard LN and Loughlin J: The role of inflammation-related genes in osteoarthritis. Osteoarthritis Cartilage 23: 1933-1938, 2015 